Revue internationale P.M.E.

Économie et gestion de la petite et moyenne entreprise

Revue

internationale

PME

\title{
Les pratiques de gestion du pharmacien titulaire pour mesurer et piloter son officine
}

\section{Grégory Reyes}

Volume 25, numéro 3-4, 2012

URI : https://id.erudit.org/iderudit/1018424ar

DOI : https://doi.org/10.7202/1018424ar

Aller au sommaire du numéro

Éditeur(s)

Presses de l’Université du Québec

ISSN

0776-5436 (imprimé)

1918-9699 (numérique)

Découvrir la revue

Citer cet article

Reyes, G. (2012). Les pratiques de gestion du pharmacien titulaire pour mesurer et piloter son officine. Revue internationale P.M.E., 25(3-4), 289-316. https://doi.org/10.7202/1018424ar
Résumé de l'article

L'officine française est plongée dans un climat d'incertitude face aux évolutions conjoncturelles auxquelles elle est confrontée (concurrence, réglementation, harmonisation européenne). Dans un tel contexte, elle semble de plus en plus exprimer le besoin de maîtriser et piloter son activité. Cette recherche s'inscrit dans cette perspective en étudiant les pratiques de gestion de quatre officines. En partant de la littérature sur la TPE (très petite entreprise), ce travail insiste sur l'influence du dirigeant-propriétaire sur les pratiques de gestion pour mesurer et piloter l'officine. 


\title{
Les pratiques de gestion du pharmacien titulaire pour mesurer et piloter son officine
}

Grégory REYES

IAE de Poitiers

\author{
MOTS CLÉS
}
Pharmacien d'officine - Dirigeant-propriétaire - Vision stratégique
Pratique de gestion - Contrôle de gestion - TPE

\begin{abstract}
L'AUTEUR
GRÉGORY REYES est maître de conférences à l'Institut d'administration des entreprises - IAE de Poitiers. II consacre ses activités de recherche à l'étude des PME sur des problématiques stratégiques et organisationnelles d'aide au pilotage, d'outils de gestion et de design organisationnel. II s'intéresse plus particulièrement aux évolutions de la pharmacie française et aux conséquences que cela entraîne sur sa gestion. Adresse: IAE de Poitiers, CEREGE, 20, rue Guillaume-VII-le-Troubadour, B.P. 639, 86022 Poitiers, France. Courriel: <greyes@iae. univ-poitiers.fr>.
\end{abstract}

\section{RÉSUMÉ}

L'officine française est plongée dans un climat d'incertitude face aux évolutions conjoncturelles auxquelles elle est confrontée (concurrence, réglementation, harmonisation européenne). Dans un tel contexte, elle semble de plus en plus exprimer le besoin de maitriser et piloter son activité. Cette recherche s'inscrit dans cette perspective en étudiant les pratiques de gestion de quatre officines. En partant de la littérature sur la TPE (très petite entreprise), ce travail insiste sur l'influence du dirigeant-propriétaire sur les pratiques de gestion pour mesurer et piloter l'officine.

\begin{abstract}
The French chemist's is plunged into state of uncertainty in the face of the economic evolution (competition, regulations and European harmonization) it is confronted with. In such a context, it appears to be expressing more and more the need to control and manage its activity. This research work comes within the scope of this
\end{abstract}


perspective through a study of the management practices of four different chemist's. Based on the literature about VSE (very small enterprise) this work emphasizes the influence of the owner-manager in the management practices to assess and run its dispensary.

\section{RESUMEN}

Las farmacias franceses están sumidos en un clima de incertidumbre ante las evoluciones coyunturales a las que se confrontan (competencia, reglamentación, armonización europea). En este contexto necesitan controlar y dirigir cada vez más sus actividades. Esta investigación se inscribe en esta perspectiva estudiando las prácticas de gestión de cuatro farmacias. Tomando como base los trabajos sobre la microempresa, este trabajo pone de relieve la influencia del director-propietario en las prácticas de gestión para evaluar y dirigir el laboratorio.

\section{Introduction}

Le marché français de la santé est en plein bouleversement (Tabuteau, 2010). Les changements sont importants pour les usagers et sans doute radicaux pour les praticiens. En effet, le rythme soutenu des réformes du système de soin français perturbe l'exercice du métier des professionnels de santé (Noguera et Lartigau, 2009). Le canal de distribution du médicament n'est pas épargné. L'un de ces professionnels dont la sauvegarde faisait sa particularité est particulièrement touché par ces évolutions. Le pharmacien titulaire d'officine dont il est question bénéficie d'un monopole sur la dispensation du médicament qui crée un marché fermé et le protège pour le moment. Cependant, les pouvoirs publics durcissent les réformes sur les médicaments remboursés, laissent la concurrence se développer sur les produits hors médicament et commencent à vouloir reconsidérer le monopole du pharmacien sous la pression des grands distributeurs (Reyes, 2011). Dès lors, la rentabilité de l'officine ne fait que baisser (Impact pharmacien, 2009). L'exercice du métier de pharmacien titulaire d'officine est de plus en plus difficile au regard d'un statut hybride où il est à la fois un professionnel de santé et un commerçantchef d'entreprise (Debarge, 2011). Sa rémunération ne dépend que de son activité commerciale, car l'acte pharmaceutique n'est pas rémunéré.

Dès lors, ce dirigeant-propriétaire de TPE doit porter une attention toute particulière pour gérer son activité dans un environnement des plus incertains. Pour qualifier la TPE et insister sur ses spécificités, la proximité est le critère régulièrement retenu (Torrès, 2003, 2009; Marchesnay, 2008; Jaouen, 2008). Ce management de proximité dont ces entreprises font preuve (Torrès, 2003) explique particulièrement l'attitude du dirigeant (Torrès, 2009; Jaouen, 2010). Cette proximité pourra être un réducteur d'incertitude pour 
le dirigeant (Torrès et Guéguen, 2008). Elle explique l'importance accordée à sa vision stratégique comme déterminant de ses choix d'organisation et de ses pratiques de gestion (Jaouen et Tessier, 2008). Le caractère psychologique du dirigeant par ses intentions et sa vision du marché explique son attitude. Devant le durcissement de son environnement, il exprime souvent un besoin en outils de mesure et de pilotage de son entreprise (Cappelletti et Khouatra, 2009; Sandino, 2007; Germain, 2006; Nobre, 2001). Ces pratiques s'inscrivent dans un processus de management destiné à accompagner, faciliter ou permettre la mise en œuvre de la stratégie de l'organisation. Ainsi, la problématique est la suivante: Quelle est l'influence du pharmacien titulaire sur les pratiques de gestion de l'officine? Elle s'articule autour des facteurs d'influence sur les pratiques de mesure et de pilotage des officines.

Ainsi, il est supposé que la principale source d'influence en TPE est le dirigeant-propriétaire et sa vision de l'environnement (Jaouen, 2010). À partir de la typologie de Jaouen (2010) sur les profils de dirigeants-propriétaires en TPE, il sera possible de définir les types de titulaires des quatre officines étudiées pour ensuite déterminer l'influence de ses dirigeants sur les pratiques de gestion de ces entreprises.

La première partie exposera le cadre théorique mobilisé. Il correspond aux travaux sur le dirigeant de TPE portant sur ses caractéristiques, notamment sa vision stratégique. Par ailleurs, une analyse sur les pratiques de mesure et de pilotage en PME est proposée afin de l'utiliser comme grille de lecture pour faire le lien entre profils et pratiques du dirigeant. La deuxième partie proposera la méthodologie retenue. L'étude de cas est choisie afin de permettre dans une démarche exploratoire de saisir tous les traits de caractère des dirigeants étudiés. Les résultats seront exposés dans une troisième partie. Les profils identifiés des pharmaciens titulaires au regard de la typologie de Jaouen (2010) seront proposés pour ensuite analyser leurs influences sur les pratiques de gestion de ces officines.

\section{Cadrage conceptuel}

Il est difficile de cerner l'objet TPE ${ }^{1}$ en raison de ses nombreuses formes et évolutions. Marchesnay a dénoncé un certain nombre d'idées reçues à son sujet en montrant comment ce type d'entreprise, en raison de sa diversité, pouvait être polymorphe $(2003,2008)$. Cependant, il est possible de leur

1. La recommandation européenne du 3 avril 1996 (mise à jour le 6 mai 2003) définit la TPE comme une entreprise dont l'effectif salarié est de moins de 10 personnes, et dont le chiffre d'affaires et le total du bilan atteignent au plus 2 millions d'euros. 
trouver des caractéristiques communes grâce à une lecture par le prisme de la proximité (Torrès, 2003, 2009; Torrès et Gueguen, 2008). La proximité est au cœur des pratiques managériales des TPE (Jaouen, 2008). En effet, les spécificités de gestion à l'œuvre dans ce type de structure peuvent être réaffirmées selon le prisme de la proximité (Torrès, 2003). La TPE fait preuve d'une proximité géographique en étant proche de ses partenaires (clients et fournisseurs). Elle a un système d'information de proximité basé sur une proximité relationnelle (interne et externe) ainsi qu'une proximité fonctionnelle correspondant à une faible spécialisation des tâches. Enfin, l'une de ses caractéristiques importantes est la proximité hiérarchique (centralisation de la gestion) indiquant l'omniprésence du dirigeant dans ce type d'entreprise (Jaouen et Tessier, 2008).

\subsection{Les caractéristiques du dirigeant-propriétaire}

Afin d'affirmer l'importance du rôle du dirigeant-propriétaire ${ }^{2}$ en TPE, Torrès va jusqu'à parler de «management sensoriel». «Dans une TPE, il est possible pour un dirigeant de "toucher" physiquement, voir, sentir, entendre... son personnel, ses clients, ses fournisseurs » (Torrès, 2009, p. 352). Dès lors, plusieurs caractéristiques peuvent lui être attribuées. Il entretient une relation particulière avec son entreprise. Cette relation dialogique au sens de Fonrouge (2002) fait coexister les logiques de l'individu et celles de l'entreprise. Ces dernières correspondent à un projet qui s'inscrit dans un projet plus global de vie propre au dirigeant. L'entreprise est alors un mode de réalisation des objectifs personnels. Dès lors, elle devient le reflet du dirigeant par ses choix stratégiques et les méthodes sélectionnées pour agir. L'activité de l'entreprise dépendra largement de la capacité du dirigeant à traiter et à exploiter l'information disponible pour prendre des décisions (Jaouen, 2010); ses sens lui sont alors indispensables pour capter des informations (Torrès, 2009). Cette proximité sera un réducteur d'incertitude (Torrès et Guéguen, 2008). La complexité et l'incertitude du marché ne sont pas des situations propres aux TPE. Cependant, le dirigeant-propriétaire, en raison d'une gestion centralisée, une stratégie intuitive et peu formalisée, n'est pas des mieux armé pour résoudre ces situations (Schmitt, Julien et Lachance, 2002).

La dimension cognitive du dirigeant sera donc essentielle dans sa représentation de l'environnement. En raison des limites évoquées, il a beaucoup de difficultés à lire son environnement et à positionner son entreprise sur

2. Il faut distinguer le propriétaire-dirigeant de l'entrepreneur. Le premier est à la tête d'une entreprise dont il est à la fois le principal décideur et le propriétaire. Le second caractérise un individu qui peut être à l'origine d'une organisation, un ameneur d'affaires, un créateur de valeur ou un innovateur (Jaouen, 2010). 
le marché (Silvestre et Goujet, 1996). L'incidence sur sa démarche stratégique sera importante. Silvestre et Goujet (1996) expliquent que lorsque l'environnement est perçu comme illisible, la dimension qualitative et la part des représentations cognitives s'imposent pour positionner l'entreprise sur le marché. En TPE, il n'est pourtant pas seul pour prendre des décisions; Marchesnay (2003) souligne le rôle de ses proches pour l'accompagner dans le développement de l'entreprise et ses choix stratégiques.

Ces caractéristiques du dirigeant-propriétaire ont inspiré de nombreuses typologies sur la $\mathrm{PME}^{3}$. Elles ont pour la plupart les mêmes bases, les caractéristiques personnelles et psychologiques du dirigeant, et conduisent à s'interroger sur les objectifs qu'il poursuit et les comportements qu'il adopte (Paradas, 1996).

La typologie de Smith (1967) est considérée comme la plus ancienne. Elle a comme principal intérêt d'associer les caractéristiques propres d'un individu à un style de gestion afin d'établir des profils types. À partir de cette contribution, de nombreux chercheurs ont repris cette approche dans leurs études. Ces différents profils sont des idéaux-types qui, comme le relève Marchesnay (1992), doivent être recoupés avec des variables contingentes pour nuancer le portrait. Il en retient plusieurs, comme l'influence du milieu social, l'éducation, l'expérience antérieure et les variables psychologiques de l'individu. Par ailleurs, plusieurs auteurs considèrent que le profil des dirigeants n'est pas figé, il évoluera d'une catégorie à une autre en fonction de sa maturité.

En TPE, compte tenu des caractéristiques attribuées au dirigeantpropriétaire, notamment le caractère subjectif de ses représentations lors de sa prise de décision, le concept de vision suscite l'intérêt (Smida et Condor, 2002; Bayad et Garand, 1998; Cossette, 1996; Carrière, 1990). Ces travaux ont mis en évidence plusieurs relations, notamment entre la vision et le processus décisionnel. Ainsi, dans une perspective dynamique, Carrière définit la vision comme: «une construction mentale d'un futur souhaité et possible pour une entreprise» (1990, p. 304). De cette vision de l'individu et de sa perception de la réalité vont découler la stratégie et son action. La vision est la représentation d'un avenir à construire.

La vision stratégique est alors la représentation d'un changement organisationnel et/ou environnemental dans un avenir lointain (Smida et Condor, 2002). Cependant, cette vision sera dépendante des valeurs et aspirations personnelles du dirigeant.

3. Pour une présentation détaillée de ces typologies, voir Messeghem et Sammut (2011, p. 60). 
Cette vision transformée en action prend forme autour d'un système de relations, tant internes qu'externes, au sein duquel le propriétaire-dirigeant développe ses apprentissages, structure ses références cognitives, définit ses rôles et échange ses visées commerciales et technologiques avec d'autres entrepreneurs, gestionnaires et représentants d'organisation de toute dimension (Bayad et Garand, 1998, p. 1).

En exploitant le concept de vision, Jaouen (2010) propose de dépasser les typologies classiques basées sur une approche comportementale du dirigeant pour se concentrer sur les aspects psychologiques de celui-ci. Elle établit quatre profils de dirigeant coexistant en TPE en fonction de leur vision et de leurs objectifs. Elle positionne ses profils sur deux dimensions. La première concerne la vision établie sur un continuum opposant l'affectif et l'émotion à la vision traditionnelle et réfléchie. La seconde correspond à la perception de l'environnement d'un côté, craint et subi et, de l'autre, du continuum favorable et rempli d'opportunités. Ces profils types sont synthétisés dans le tableau 1.

Cette analyse ne se concentre pas directement sur les stratégies des entreprises, mais sur les visions qui leur sont sous-jacentes. À partir de ces profils de dirigeant et de leur vision, il sera intéressant d'associer les pratiques de gestion pour mesurer et piloter leur activité compte tenu de la relation dialogique qu'entretiennent l'individu et son entreprise (Fonrouge, 2002).

\subsection{Pratique de gestion en PME pour mesurer et piloter l'activité}

Le dirigeant-propriétaire en PME est le premier déterminant de la performance de son entreprise (Reijonen et Komppula, 2007). Cependant, il est vite contraint dans ses choix lorsqu'il fait face à un environnement dynamique (Silvestre et Goujet, 1996). Dès lors, les outils de gestion lui permettront d'améliorer son processus décisionnel (Bayad, Gallais et Schmitt, 2006). Ainsi, en évoquant la mesure et le pilotage de la performance de l'entreprise, le terme «management control», comme notion étendue du contrôle de gestion, prend tout son sens.

Il est pourtant difficile en TPE de parler de contrôle de gestion. Cappelletti et Khouatra (2009) constatent que les petites structures sont souvent dotées d'un système de contrôle rudimentaire. Germain (2006) souligne la «rusticité » des outils de mesure en PME. Le système de contrôle de gestion se structurera avec l'augmentation de la taille de l'entreprise (Van Caillie, 2003 ; Dávila et Foster, 2005 ; Sandino, 2007). Plusieurs auteurs considèrent que les buts des dirigeants sont des éléments moteurs dans le choix des modes opératoires de la mesure et du pilotage de l'entreprise (Nobre, 2001; Van Caillie, 2003; Condor et Rebut, 2008; Meyssonnier et Zawadzki, 2008; 


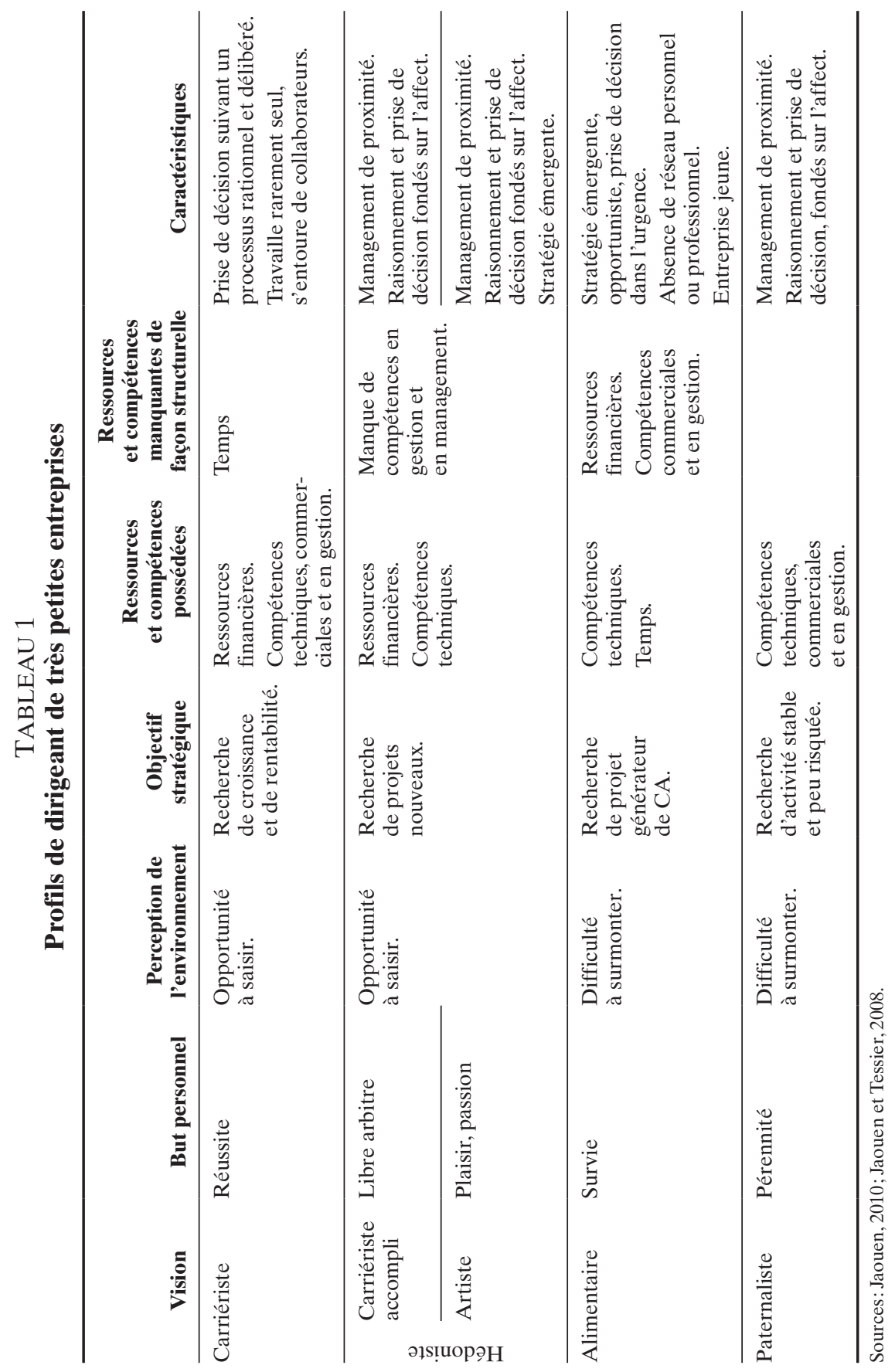


Cappelletti et Khouatra, 2009). Le chef d'entreprise sera même le garant de la réussite de l'introduction d'un système de mesure et de pilotage en PME s'il fait preuve d'une attitude volontariste (Meyssonnier et Zawadzki, 2008; Cappelletti et Khouatra, 2009). Condor et Rebut (2008), pour analyser les pratiques de contrôle en PME, utilisent huit items: la mission du contrôle de gestion, les acteurs, les méthodes utilisées, les systèmes d'information utilisés, la périodicité du contrôle, l'horizon des prévisions, l'externalisation de certaines pratiques et la diffusion des résultats. En reprenant certains de ces items, il est possible de faire plusieurs constats.

Ainsi, on notera que la mission principale du contrôle de gestion en PME est le calcul des coûts et des prix (Nobre, 2001; Van Caillie, 2003). Cappelletti et Khouatra (2009) affirment qu'un système de contrôle de gestion adapté en PME est un vecteur d'amélioration des performances de l'entreprise.

Il permet donc de mesurer, mais également de piloter la performance de ces entreprises. Ce pilotage pourra également s'organiser par l'intermédiaire de tableaux de bord (Germain, 2006).

Les acteurs majeurs du contrôle de gestion dans les PME sont les cadres financiers et les dirigeants (Nobre, 2001; Van Caillie, 2003). Le degré de spécialisation de la fonction augmente avec la taille de l'entreprise. Dans les petites structures, c'est généralement le propriétaire-dirigeant qui assume cette charge (Sandino, 2007; Cappelletti et Khouatra, 2009).

Concernant les méthodes utilisées, différentes analyses montrent que c'est le calcul des coûts, notamment sur le suivi des marges par produit qui est le plus utilisé (Nobre, 2001; Van Caillie, 2003; Condor et Rebut, 2008). Le contrôle budgétaire ressort également des analyses avec une élaboration annuelle et un suivi régulier des écarts (Nobre, 2001; Van Caillie, 2003; Dávila et Foster, 2005; Condor et Rebut, 2008); par contre, le tableau de bord est encore un outil peu utilisé par rapport aux deux autres méthodes (Nobre, 2001; Van Caillie, 2003; Germain, 2006). Il semble qu'en PME il reste encore assez artisanal et peu stratégique, car focalisé sur des indicateurs essentiellement financiers et comptables (Germain, 2006).

La taille semble être un facteur déterminant dans l'utilisation d'un système d'information comptable (Condor et Rebut, 2008). En effet, les TIC peuvent être dans les PME des sources d'avantages concurrentiels simultanément exprimables en termes de réduction des coûts, d'amélioration de la qualité et d'innovation de produits et services (Amabile et Gadille, 2003). Cependant, Boutary constate que: «les TPE intègrent insuffisamment les TIC et en réservent l'usage à une extension de leur terrain d'action, sans remise en cause profonde de leur mode de gestion» $(2008$, p. 75$)$. 
En définitive, il semble que la loi proxémique de Torrès (2003) sur les spécificités de la PME, explique certaines pratiques en raison du faible espace dans lequel elles s'exercent. C'est d'autant plus significatif dans les TPE où, selon un effet de grossissement des problèmes à gérer, le phénomène est accentué (Mahé de Boislandelle, 1996). Le dirigeant-propriétaire sera alors le principal initiateur et utilisateur des pratiques de gestion pour mesurer et piloter son activité. Cependant, proximité ne veut pas dire systématiquement simplicité ou absence de pratique.

\section{Design de la recherche}

L'objectif de cette recherche, en analysant les officines françaises, est de s'interroger à propos de l'influence du dirigeant sur les pratiques de gestion de ces TPE. Ce travail sert donc à analyser le «pourquoi» et le «comment» de telles pratiques dans ces entreprises (Wacheux, 1996). La démarche par étude de cas a une visée compréhensive et contextualisée dans le sens où les comportements humains et organisationnels ne peuvent se comprendre et s'expliquer qu'en relation avec les significations que les personnes donnent aux choses et à leurs actions (Hlady-Rispal, 2002). L'étude de cas permet notamment de se concentrer sur l'examen des processus décisionnels, l'élaboration et la mise en œuvre des actions organisationnelles (Yin, 2008). Pour analyser les officines dans le cadre d'une démarche exploratoire, quatre cas ont été retenus. Wacheux (1996) explique qu'un faible nombre de cas est suffisant lorsqu'il s'agit d'explorer des pratiques ou des phénomènes nouveaux. Dans le cadre d'une approche exploratoire par étude de cas, Eisenhardt (1989) situe leur nombre entre quatre et dix pour des raisons de volume des données. Cela correspond également à un principe d'équilibre (Hlady-Rispal, 2002). Le nombre de cas a été déterminé par la volonté d'obtenir une variété équilibrée de situations différentes, ici sélectionnée selon l'implantation géographique. Pour un commerce de détail, le lieu d'implantation a une incidence importante sur la configuration de l'entreprise, son activité et ses relations avec le client (Badot et Lemoine, 2010). Le grossiste répartiteur Alliance Healthcare (2009) classe les officines en quatre catégories : l'officine rurale (OR), l'officine de quartier (OQ), l'officine de centre-ville (OC) et l'officine de centre commercial (OCC). Elles se distinguent à partir de trois critères clés: le type de clientèle, les produits proposés et les charges assumées. Cette classification est reprise par l'ensemble de la profession (notamment le conseil de l'Ordre des pharmaciens). Elle reflète un élément important lié à la distribution du médicament qui est le maillage démographique impliquant une bonne répartition des officines sur le territoire. 


\subsection{Le contexte des cas: le marché de la santé}

De nombreux bouleversements touchent actuellement le secteur français de la santé (Tabuteau, 2010). À ce titre, l'activité du pharmacien est confrontée à des mutations externes importantes. Leurs impacts sont lourds de conséquences sur la gestion de leur activité en dépit de certains atouts qui font leur spécificité dans le canal de distribution du médicament (Reyes, 2011; Moinier, 2009).

Ainsi, le pharmacien a toujours bénéficié d'un avantage lié à sa situation de monopole de dispensation des médicaments fondé sur l'expertise et la responsabilité du titulaire (Déclaration du Roi, 1777). Cette situation repose sur les produits nécessitant un conseil. Selon les cas, le médicament peut représenter environ $85 \%$ à $95 \%$ du chiffre d'affaires (Rapport de la Cour des comptes sur le financement de la sécurité sociale, 2008) correspondant aux produits dont la marge est la plus faible (6\% sur certains médicaments vignettés, par exemple). C'est avec les activités hors monopole comme la parapharmacie, les produits vétérinaires, le maintien à domicile (matériel médical) que le pharmacien réalisera des marges commerciales plus importantes. La référence sur la répartition du chiffre d'affaires d'une officine est $80 \%$ pour le médicament et $20 \%$ pour les produits hors médicament. Cependant, cette répartition est très variable selon l'implantation et le type de clientèle.

Il bénéficie également d'une liaison entre le capital et l'exploitation de l'activité afin de garantir l'indépendance des actes professionnels. Pour éviter tout risque relatif à des pressions financières externes, il est le seul pouvant être propriétaire d'une officine et répondre d'un service de santé publique. Enfin, le maillage démogéographique pour un accès équilibré est également un avantage sur le marché. En effet, l'installation des officines et leur répartition sont limitées par un numerus clausus (une officine pour environ 3000 habitants). C'est la garantie pour la population d'une présence géographique équitable en termes d'accès aux médicaments et d'une bonne régulation de la concurrence. Aujourd'hui, ces atouts peinent à résister aux évolutions du secteur. Le tableau 2 en synthétise les principales.

Les conséquences de tous ces éléments se répercutent sur la rentabilité des officines françaises. Ce commerce n'est plus aussi prospère et plusieurs officines arrêtent leur activité. En effet, on dénombre au

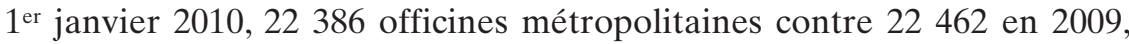
soit une variation de $-0,34 \%$ (site Internet de l'Ordre des pharmaciens, 2011). Devant ce constat, le pharmacien déploie beaucoup d'efforts pour renforcer son rôle de gestionnaire d'entreprise (Impact Pharmacien, 2009). Le paradoxe de ce métier réside dans la non-reconnaissance de l'acte du pharmacien au même titre que d'autres professionnels de santé 


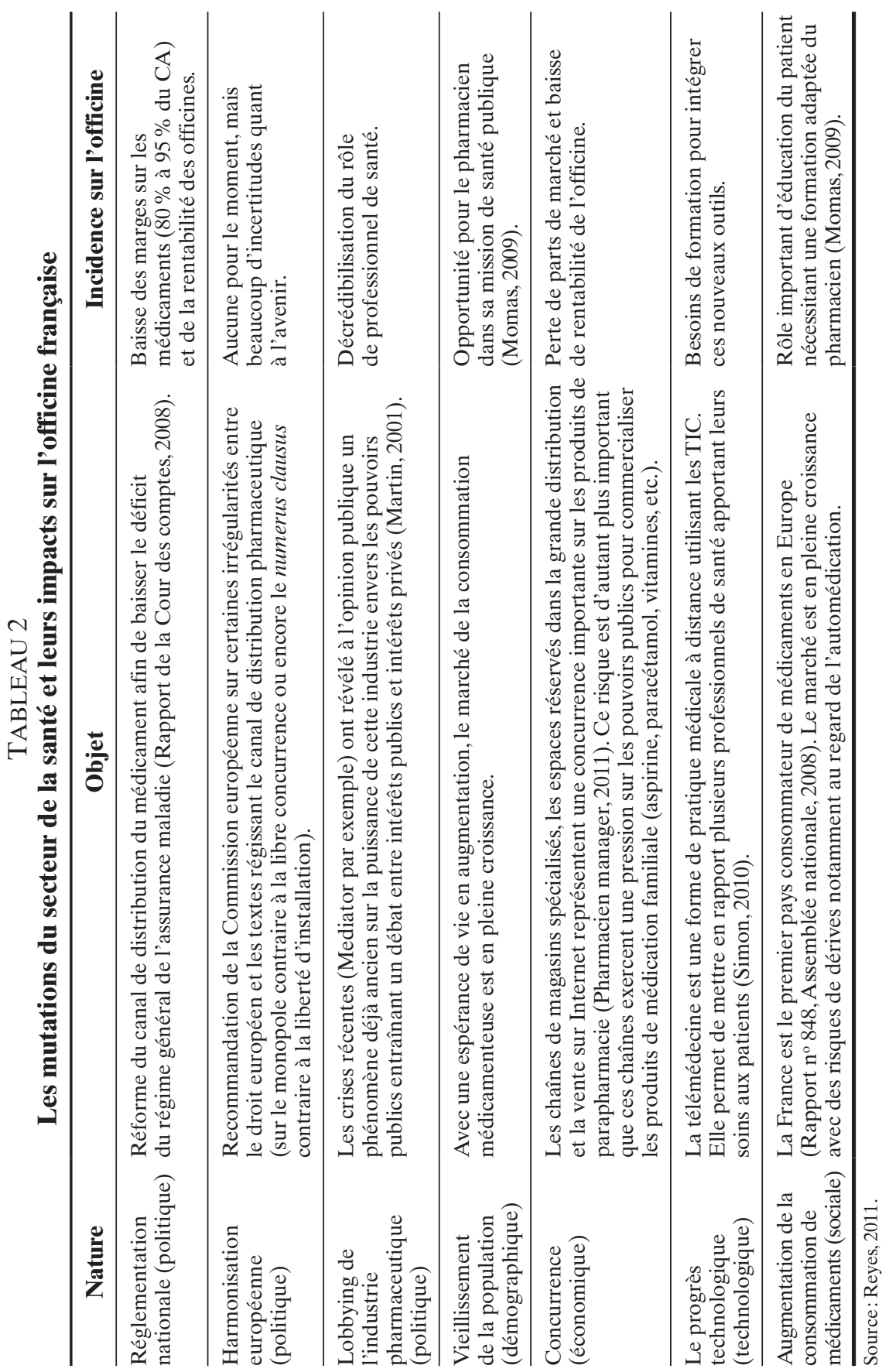


comme le médecin. Sa rémunération n'est fondée que sur la marge commerciale (Debarge, 2011). Il focalise ses efforts pour maîtriser et piloter son activité parfois avec peu de compétences en la matière (Reyes, 2011).

En effet, sa formation initiale le prépare avant tout à la connaissance des médicaments, leur délivrance et le conseil aux clients; il n'est pas ou peu formé à gérer une entreprise. Cet apprentissage se fait davantage lors de ses stages en officines ou de sa période d'assistanat devançant généralement son installation. Dès lors, le pharmacien titulaire nouvellement installé exprime vite le besoin en techniques et outils pour gérer et optimiser son activité.

\subsection{Présentation des cas}

En reprenant la typologie de l'officine du grossiste Alliance Healthcare (2009), quatre cas sont étudiés. Les officines sont toutes implantées en France dans la région Centre. Elles sont exposées en détail dans le tableau 3.

\subsection{La méthode d'analyse}

La méthode correspond à une analyse de contenu thématique du discours des différentes personnes interviewées (Bardin, 2003). Elle peut s'utiliser en sciences de gestion, notamment lorsqu'on cherche à connaître et comparer les attitudes, intentions, pratiques et croyances des dirigeants (Gavard-Perret, Gotteland, Haon et Jolibert, 2008). Compte tenu du rôle majeur du dirigeant dans la conduite et les orientations à donner en TPE (Marchenay, 2003), le pharmacien est la principale personne interviewée. Cependant, dans le cadre d'études de cas, plusieurs matériaux ont été utilisés pour collecter des informations sur les entreprises étudiées. Chaque pharmacien titulaire a été interviewé lors d'entretiens semi-directifs avec des guides d'entretien à structure faible (Blanchet et Gotman, 2005, p. 61). Par ailleurs, pour s'affranchir du travail de contextualisation, des visites régulières avec observations des pratiques en situation ainsi qu'une collecte de documents clés (outils de gestion, données comptables et financières, rapport d'activité, entretiens avec les salariés, etc.) ont été nécessaires tout au long de l'étude afin d'effectuer une triangulation des informations (Wacheux, 1996).

La démarche générale suit un protocole en trois étapes. La préanalyse, tout d'abord, correspond à une lecture flottante qui permet de déterminer des règles de découpage ainsi que l'identification et la définition des catégories pertinentes. Les dirigeants ont été amenés à répondre à des questions ouvertes sur leur vision du marché, l'exercice de leur activité, leurs pratiques de gestion. Les grilles de lecture proposées par Jaouen (2010) sur les caractéristiques du dirigeant et celles de Condor et Rebut (2008) sur les pratiques de contrôle des 


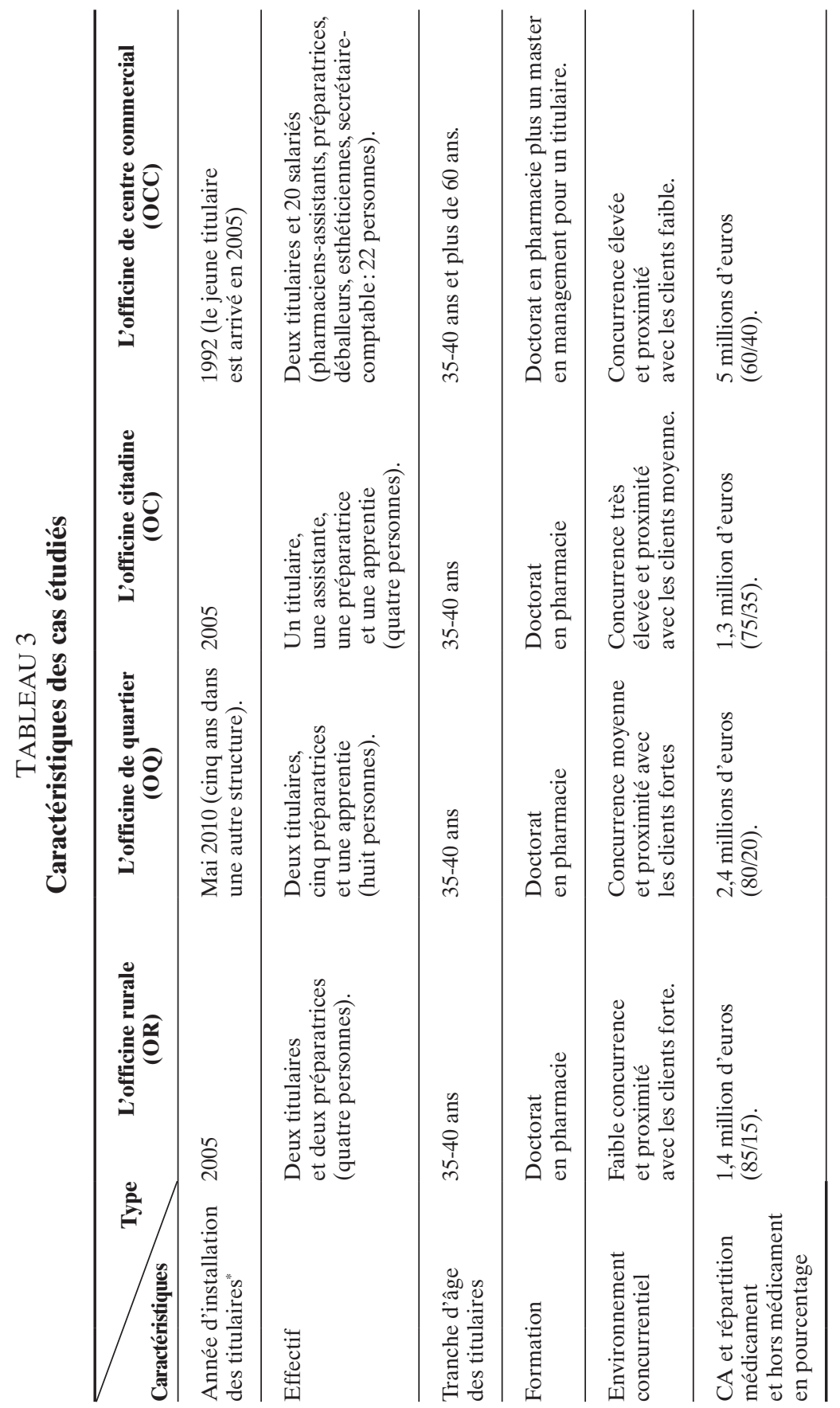




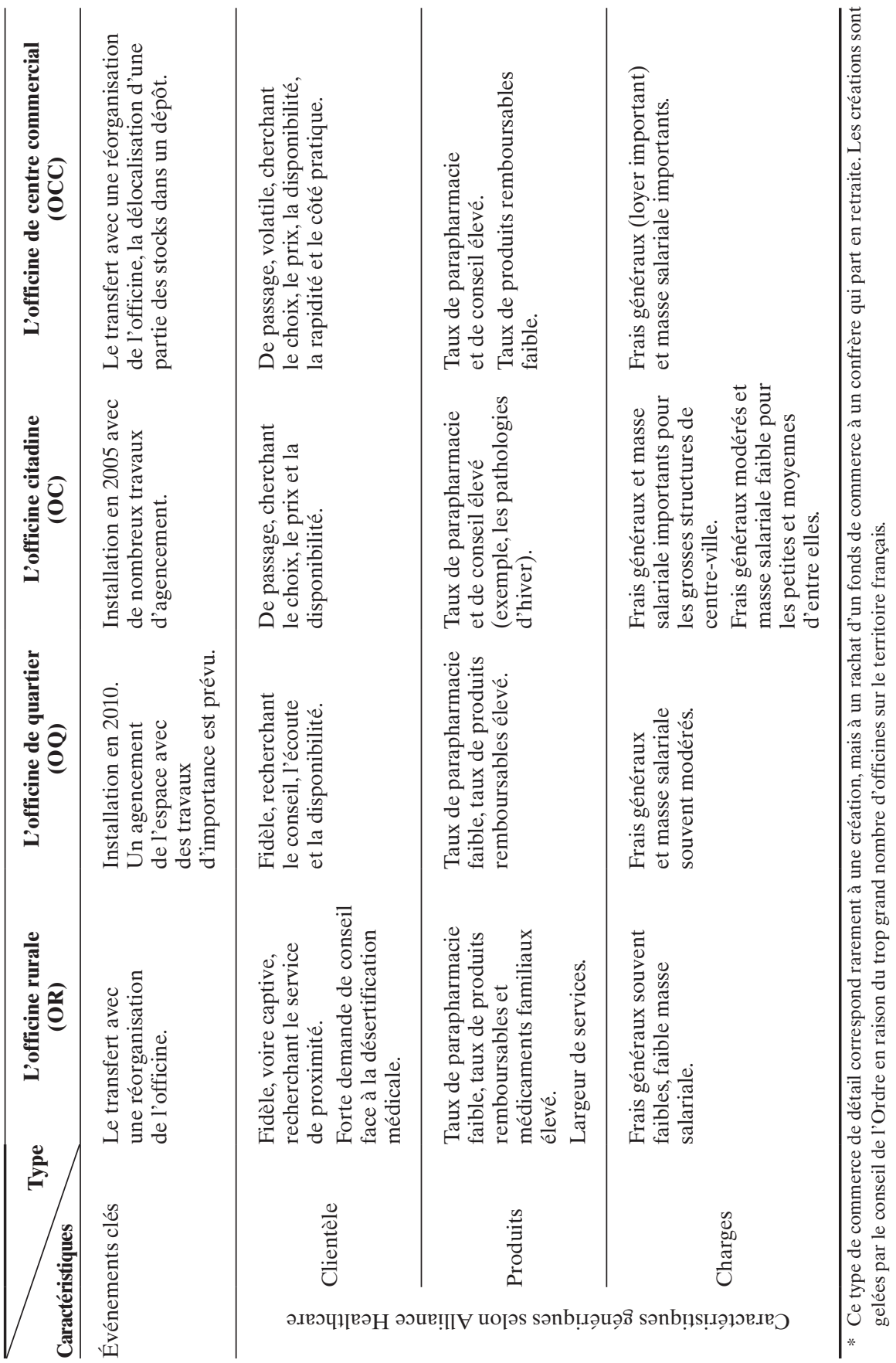


PME ont permis d'organiser et de classer le verbatim. Des inférences ont été réalisées entre les cas étudiés et la typologie de Jaouen (2010) ainsi que des interprétations sur les pratiques de gestion de ces entreprises. Cette démarche a donc permis une déconstruction où l'on détache certains éléments de leur contexte et une reconstruction où l'on propose un nouvel assemblage de données porteur d'un sens nouveau (Gavard-Perret et al., 2008).

\section{Résultats: profils des dirigeants-propriétaires et pratiques de gestion des officines}

Le pharmacien titulaire d'officine française est dans une situation assez délicate au sein du canal de distribution du médicament. L'exercice de son métier change progressivement au rythme des réformes et des évolutions du marché de la santé (Reyes, 2011). Autrefois professionnel de santé à part entière, il se souciait peu de la rentabilité de son commerce. Le marché était prospère et les contraintes peu nombreuses. La situation est tout autre aujourd'hui, car il doit endosser pleinement son rôle de dirigeant-propriétaire de TPE devant faire face à un marché complexe et incertain. Dès lors, comme toute autre TPE, les comportements du dirigeant (motivation, dynamisme, compétences, etc.) et sa vision du marché influencent les orientations stratégiques données à l'entreprise (Jaouen, 2010; Marchesnay, 2008). Par conséquent, les pratiques de gestion de ces entreprises dépendent des profils de leurs dirigeants en considérant qu'il y a coexistence entre les logiques de l'individu et celles de l'entreprise (Fonrouge, 2002).

\subsection{Les caractéristiques du pharmacien titulaire et sa vision stratégique}

La première constatation sur les quatre cas étudiés est générale. Tous les dirigeants-propriétaires ont pleinement conscience de la situation actuelle du marché de la santé et de ses incidences sur le canal de distribution du médicament et, plus précisément, sur l'avenir de leur métier. Le sentiment global sur cette perception de leur environnement reste assez pessimiste en définitive même si certains trouvent le moyen de développer leur métier. Il ressort de leur discours le sentiment d'une grande incertitude sur ce marché.

«Notre marge est de plus en plus rognée. Nous ne savons pas vraiment où nous allons» (OQ). "C'est de plus en plus dur. On doit multiplier les efforts pour maintenir sa rentabilité» (OC). «Nous sommes dans une situation précaire, car nos marges diminuent, nous avons le sentiment que le but est d'asphyxier le maximum d'officines»(OR). «Financièrement, c'est de plus en plus serré à cause des marges qui diminuent. On sera sûrement obligé de faire évoluer notre métier» (OCC). 
Malgré cette perception de l'environnement, certains, sans y voir de totales opportunités, distinguent de possibles évolutions de leur métier. L'OR et l'OQ par exemple, en s'appuyant davantage sur leur relation de proximité avec le client, pensent à un renforcement de leur rôle de professionnel de la santé. En s'appuyant sur le dispositif prévu par la loi Hôpital, Patient, Santé et Territoire (Loi HPST du 21 juillet 2009), dont le but est notamment d'assurer une meilleure coordination entre les professionnels de santé, ils pensent revaloriser cette facette de leur métier. En effet, selon l'article 38 de cette loi, le pharmacien pourra prendre en charge les soins de premier recours, devenir le pharmacien référent en établissement d'hébergement des personnes âgées, participer à l'éducation thérapeutique et aux actions d'éducation des patients.

Ce statut de pharmacien «correspondant» jette les bases d'une revalorisation de l'acte pharmaceutique (Le pharmacien de France, 2011).

L'OCC dans sa situation ressent surtout le besoin de développer son activité pour absorber les contraintes du marché. "Pour survivre, une pharmacie devra grossir parce que les coûts de fonctionnement sont tels que la seule façon de compenser est d'augmenter son activité et son chiffre» (OCC); ce scénario n'est cependant pas réalisable pour tous. Si le centre commercial amène suffisamment de passage à l'OCC, ce n'est pas le cas pour l'OC. Cette dernière est petite pour sa catégorie et elle est très fortement concurrencée par deux autres officines à quelques mètres dans la même rue. Dès lors, son objectif principal est le maintien de son activité. "Quand je dis que j’ai une vision étroite, j'essaie déjà de ne pas perdre d'argent. Pour en gagner plus, je n'ai pas forcément le temps de me tourner vers l'aspect gestion»(OC). Ainsi, à partir de cette perception de l'environnement, les dirigeants des quatre officines expriment différents objectifs stratégiques. L'origine de ces choix vient en partie de la source de l'avantage concurrentiel qu'ils désirent exploiter. L'OR et l'OQ jouent avant tout sur le rôle de proximité en assurant pleinement leur mission de santé publique. Elles se rendent très disponibles et prennent du temps pour dialoguer avec leurs clients, apportent des soins de premier recours et des conseils avisés. L'OC favorise l'accueil et le conseil dans une perspective de fidélisation pour faire face à une forte concurrence de proximité. L'OCC orientera son avantage concurrentiel sur la disponibilité de ses produits, le temps d'attente et le prix, car son activité dépend avant tout de la fréquentation de la galerie. Son objectif est de croître afin d'atteindre une taille critique lui permettant d'assumer ses charges de structure et d'absorber les contraintes du marché. 
Eu égard à ces différents objectifs, la question des compétences du dirigeant se pose inévitablement. Comme le souligne la titulaire de l'OC, ses compétences en gestion restent limitées, car elle n'a pas été formée sur ce point. «En fait, nous ne sommes pas formés, ce n'est pas notre principal métier. Pour tout ce qui est commande et clientèle, mon métier propre, je pense avoir les compétences, mais pour la gestion, j'apprends au fur et à mesure»(OC).

Les titulaires de l'OR font le même constat. «C'est un peu l'ambigü̈té de notre travail, car on est à la fois des professionnels de santé et en plus des commerçants. Ce qui nous fait vivre, c'est notre marge commerciale. En d'autres termes, ce qu'on achète moins ce qu'on vend, sauf qu'il y a des choses que nous ne maittrisons pas» (OR).

C'est un problème récurrent pour le pharmacien d'officine, sa formation initiale comprend surtout l'acquisition de compétences techniques propres à la connaissance et la gestion des médicaments (Reyes, 2011). Cependant, si le parcours initial de formation des dirigeants étudiés est le même, un des titulaires de l'OCC a complété son apprentissage avec une formation en management dispensée par une université spécialisée. Il a alors acquis les compétences en gestion nécessaires à son projet d'officine.

À la lumière de ces éléments, le tableau 4 expose les caractéristiques des titulaires des quatre cas étudiés en reprenant la grille de synthèse sur les profils de dirigeants (Jaouen, 2010; Jaouen et Tessier, 2008).

Ce tableau montre que les profils développés par Jaouen (2010) sur la TPE s'adaptent tout à fait aux entreprises étudiées.

Ce tableau reprend les principaux traits des titulaires des officines étudiées au vu de leur perception et de leur vision du marché. Certains titulaires comme ceux de l'OR et l'OQ sont très proches, mais correspondent à un stade d'évolution différent. Les titulaires de l'OQ ont été propriétaires d'une officine rurale pendant cinq ans avant d'acquérir cette officine. C'est sans doute ce qui explique des traits de caractère très proches. Le paternaliste n'est pas représenté. Les cas d'officines familiales sont pourtant nombreux dans cette profession à l'image de l'OCC où les titulaires ont un lien mère-fils, ou encore dans l'OR où les titulaires sont mariés. Mais le rattachement des titulaires des officines aux différents profils de la typologie se fonde avant tout sur la perception de l'environnement, la vision et les objectifs stratégiques des dirigeants. Cette grille de lecture a permis d'identifier pour les quatre cas étudiés les profils de leurs dirigeants-propriétaires. Ils renseignent sur la vision stratégique des acteurs étudiés et permettent d'observer l'influence de chaque profil sur les pratiques de gestion de ces officines. 


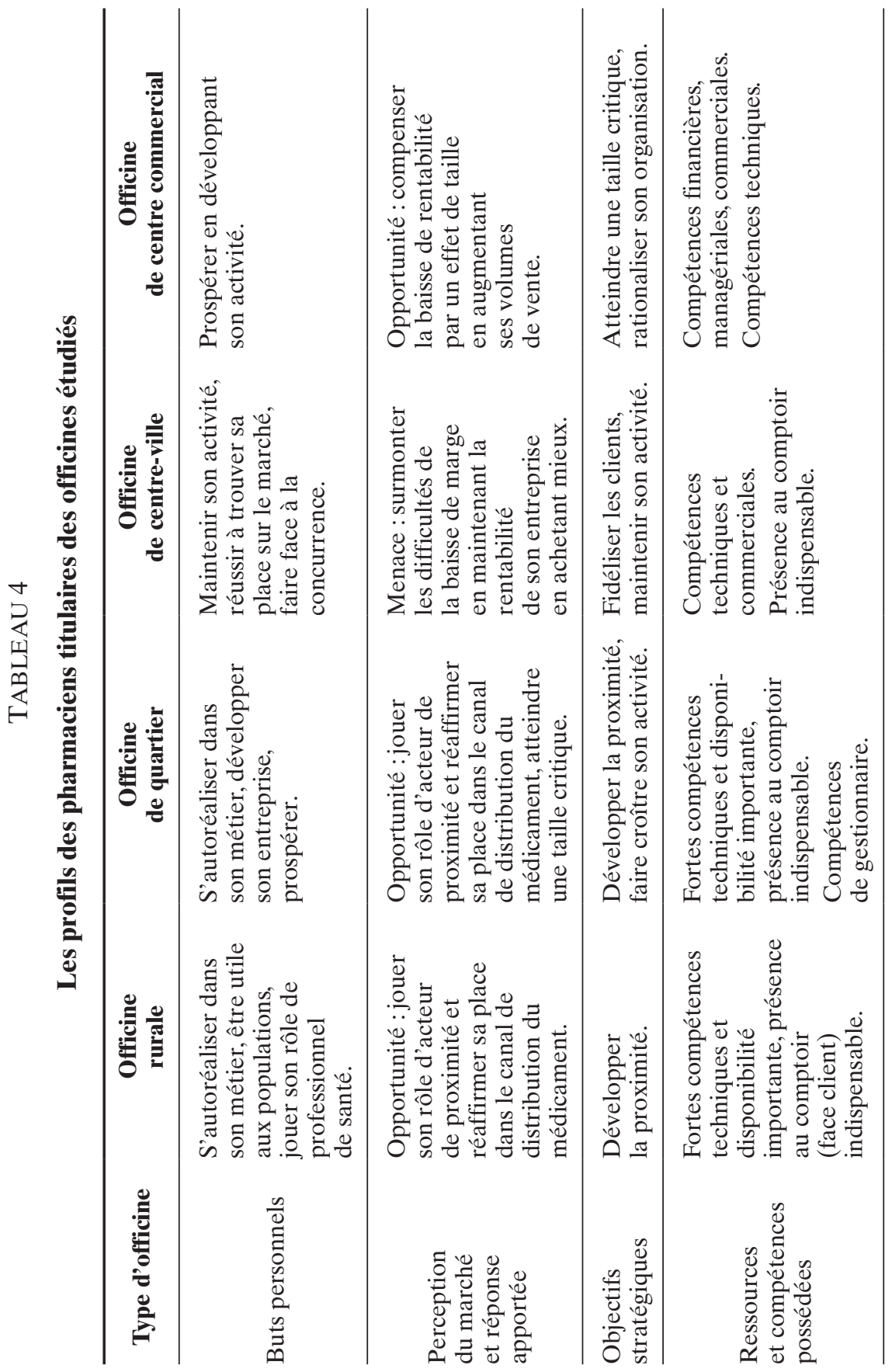




\begin{tabular}{|c|c|c|}
\hline 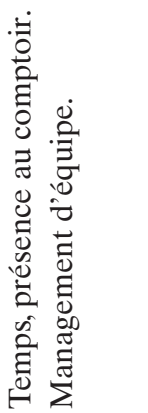 & 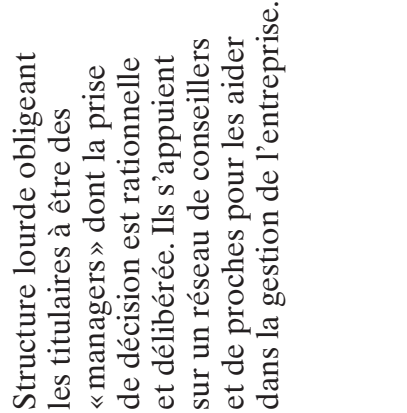 & 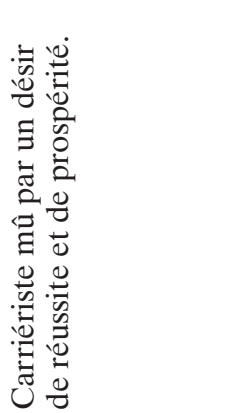 \\
\hline 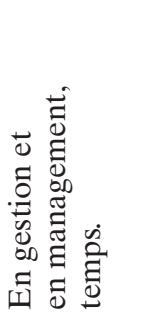 & 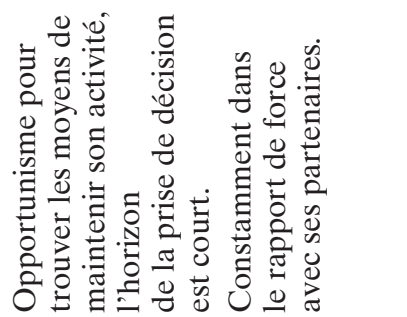 & 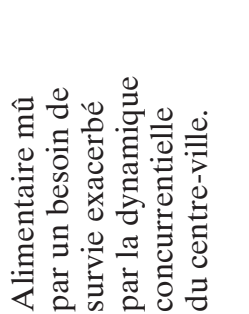 \\
\hline 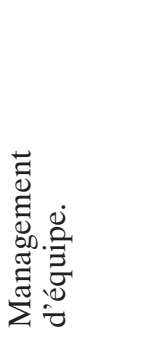 & 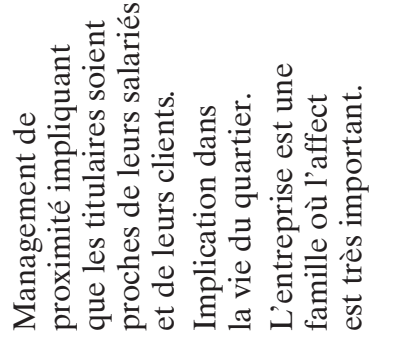 & 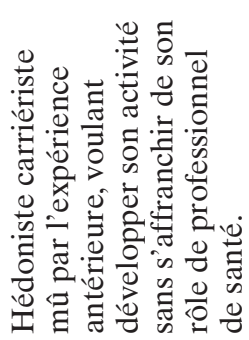 \\
\hline 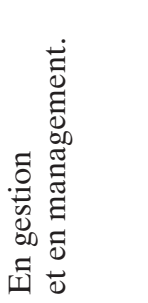 & 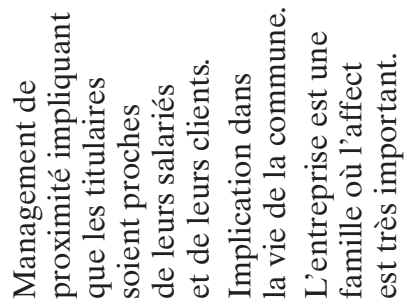 & 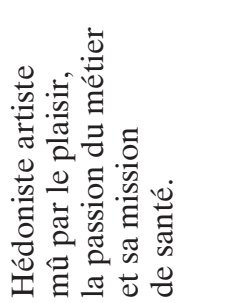 \\
\hline 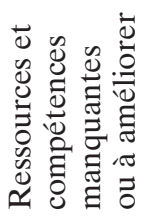 & 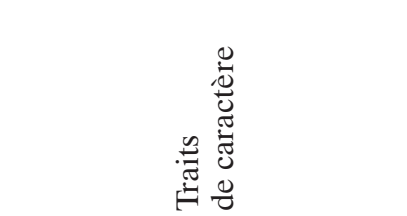 & 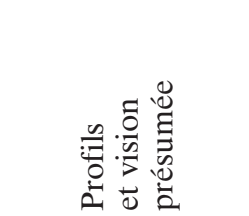 \\
\hline
\end{tabular}




\subsection{Les pratiques de gestion des pharmaciens titulaires}

Les difficultés auxquelles se heurtent les pharmaciens titulaires aujourd'hui s'expliquent par la baisse de rentabilité des officines. Les causes principales sont les réglementations de la part des pouvoirs publics sur la baisse des marges des médicaments. De ce fait, il est naturel dans la pratique au quotidien que l'attention des dirigeants se focalise sur cette marge. Le suivi de l'activité se fait quotidiennement à partir de données financières simples comme le chiffre d'affaires ou la marge dégagée. C'est un constat global, quel que soit le profil du dirigeant. Ce besoin de maîtriser la marge a des répercussions sur la mission attribuée à la mesure et au pilotage de l'activité. En effet, un élément récurrent du discours des pharmaciens étudié est de «savoir acheter». Derrière ces termes, ils expriment la volonté de bien gérer leurs approvisionnements, les prix de leurs produits et, d'une manière plus générale, leur activité.

Je vais voir chaque jour le chiffre que je fais et je vérifie l'évolution de ma marge. J'ai quand même une notion journalière de l'évolution de mon activité. On fait deux retours, savoir si l'activité est bonne et surtout savoir si nous achetons bien ou mal parce que finalement, c'est ça notre boulot. C'est intéressant, mais ça prend du temps et c'est un facteur de stress. Parce que nous sommes obnubilés par la marge et son évolution. C'est un peu une base fondamentale de notre exercice. Ce qui tient l'officine (OR).

Mais si tous s'accordent sur cette idée, ils ne le font pas de la même manière. Pour suivre son activité, l'OCC en tant que «carriériste» au sens de Jaouen (2010) s'appuie sur des outils d'aide au pilotage afin d'avoir une vision plus claire de son avenir. Les titulaires de cette officine semblent également avoir une certaine vision des évolutions du marché et tentent alors de les anticiper. «Nous partons du postulat et cela se confirme que notre taux de marge va baisser; donc si nous voulons maintenir notre rentabilité par rapport aux années précédentes, il faut absolument développer notre CA; donc, nous sommes contraints de faire du développement de CA par une politique de volume» (OCC). Ils ont la volonté de maîtriser leur développement et s'appuient alors sur des outils comme les tableaux de bord. Ils sont aussi constamment en recherche d'indicateur de contrôle pour mesurer leur progression. Dans une situation opposée, le titulaire de l'OC, en tant qu' ' alimentaire» mû par la survie, s'appuie sur un certain «feeling» selon ses termes pour mesurer et piloter son activité. «Mon indicateur, c'est moi» (OC). Par manque de temps et de compétences de gestion, elle gère au plus simple. Les outils utilisés lui semblent tout à fait satisfaisants même si elle a conscience des limites d'une telle méthode. Les titulaires de l'OR, en tant qu'hédonistes artistes mus par le plaisir, n'aiment pas être dépendants des chiffres et de leur rôle de gestionnaire. Ils ont l'impression que c'est au détriment de 
leur mission de santé. Cela se ressent alors sur leurs pratiques de gestion. «On peut toujours multiplier les outils pour gérer l'activité, mais on devient un peu esclave de ça. Plus on rentre dans l'engrenage d'optimiser, plus on se concentre sur des choses accessoires. Nous, c'est du global, ce sont de gros indicateurs, après on peut toujours affiner, mais est-ce le rôle du pharmacien d'affiner sa gestion? Je ne sais pas» (OR). Les titulaires de l'OQ ont une vision plus modérée sur le sujet. Ce sont des hédonistes carriéristes dont le projet doit allier la mission de santé et la proximité au développement de l'entreprise. «Notre rôle de professionnel de santé de proximité est important et on doit le réaffirmer, car on a de moins en moins de médecins. D'un autre côté, on gère une entreprise et nous sommes obligées d'être de vraies négociatrices, de bien gérer nos stocks et de gérer notre espace de vente»(OQ).

Ainsi, on constate que la vision des dirigeants étudiés influe sur leurs pratiques comme l'illustre le tableau 5. Il reprend notamment certains items de Condor et Rebut (2008) sur les pratiques de contrôle en PME.

L'intérêt du tableau est de tenter d'expliquer que les différences ou les similitudes constatées dans les pratiques de gestion de ces quatre officines ne s'expliquent que partiellement par les critères associés au lieu d'implantation (charge, produits, clients), comme pourrait le suggérer la typologie du grossiste répartiteur Alliance Healthecare (2009).

C'est, semble-t-il, le dirigeant et sa vision du marché associés à la façon dont il souhaite y faire face (intention) qui expliquent les disparités dans les pratiques de gestion.

Dès lors, on constate une structuration et une formalisation des pratiques de gestion en adéquation avec la vision du dirigeant. Les carriéristes (OCC) et les hédonistes carriéristes (OQ) sont ceux qui structurent le plus leurs pratiques avec notamment l'utilisation d'outils de gestion formalisés comme des tableaux de bord ou des budgets prévisionnels. À cet effet, quand les compétences manquent, ils savent s'entourer des personnes nécessaires. À l'opposé, l'alimentaire (OC) et les hédonistes artistes (OR) formalisent peu leurs pratiques, mais pour des raisons diamétralement opposées. L'OC, par manque de temps, focalise toute son attention sur la survie alors que l'OR fait le choix de ne pas aller plus loin, car cela ne correspond pas à sa vision du métier et du marché. Les outils utilisés sont donc informels à l'image des tableaux de bord. Ils suivent des informations journalières, hebdomadaires et mensuelles sans pour autant avoir besoin de les répertorier dans un tableau formalisé. C'est également le cas pour leur système de gestion informatisé. L'ensemble des officines étudiées en est équipé, mais tous ne l'exploitent pas de la même manière. Certaines ne l'utilisent que pour gérer leur stock (OR, OC), d'autres vont plus loin en l'utilisant comme un outil de «monitoring» 


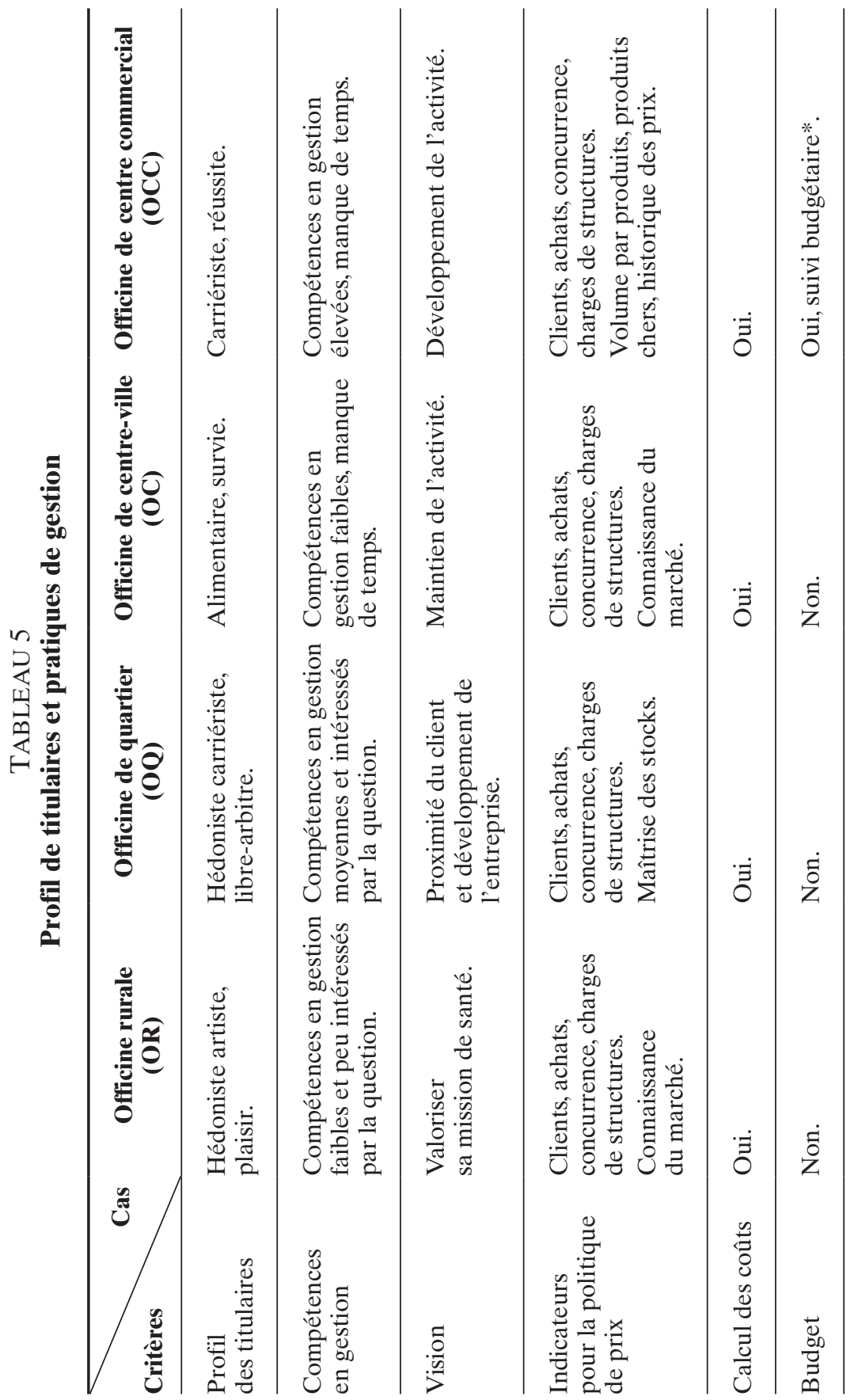

Revue internationale P.M.E., vol. 25, nos 3-4, 2012 


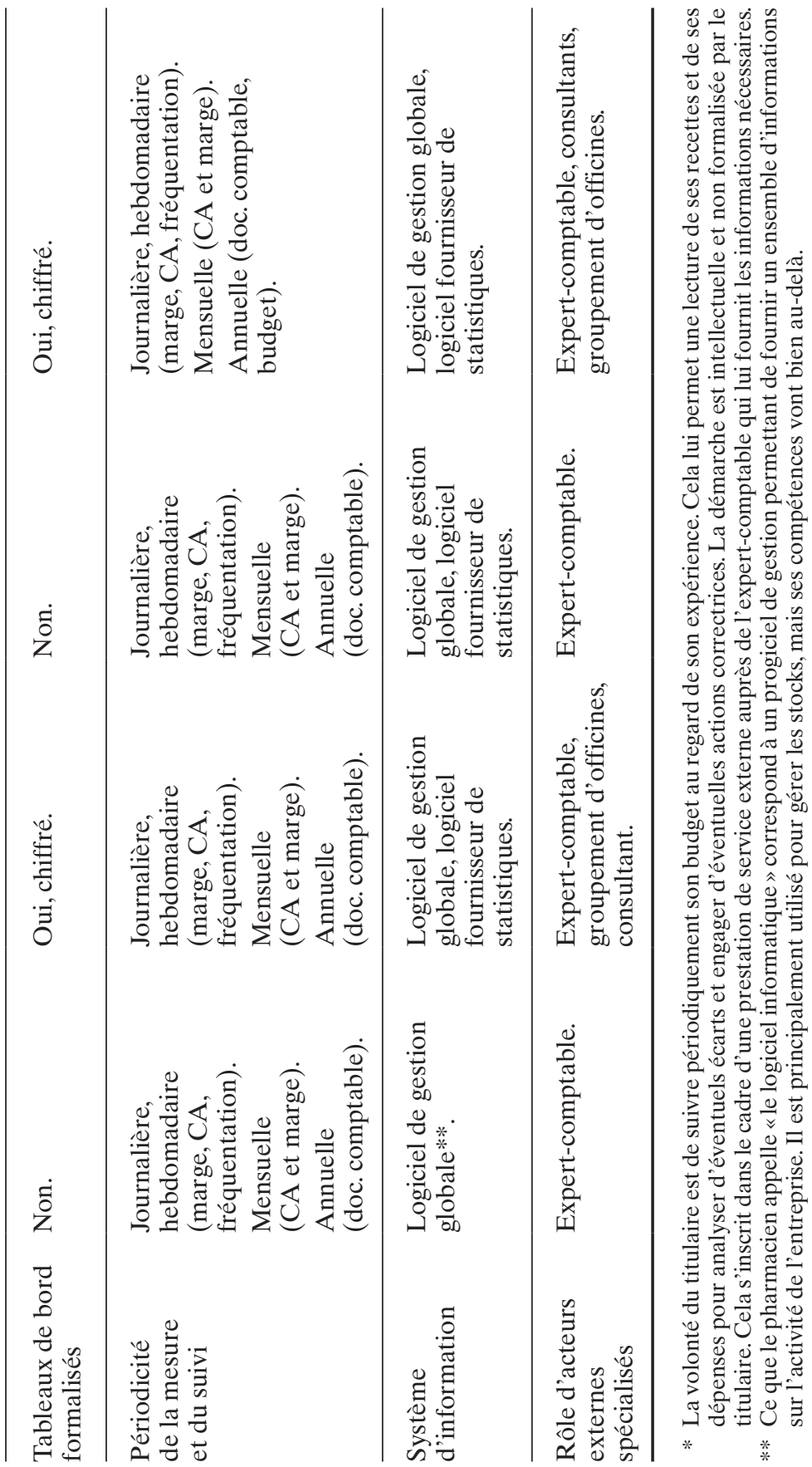


et de gestion de la relation client (OCC, OQ). Le lieu d'implantation n'est alors qu'un facteur explicatif parmi d'autres pratiques de gestion. La principale influence vient du dirigeant en fonction de sa perception des signaux du marché et la réponse qu'il leur donnera.

\section{Conclusion}

Il a été constaté que les pratiques de gestion des dirigeants des quatre officines étudiées étaient sensiblement différentes. Il y a plusieurs éléments à l'origine de ces différences. Le lieu d'implantation permet surtout d'expliquer une forme de proximité. En effet, l'OR et l'OQ sont proches de leurs clients et se rendent très disponibles selon leurs desiderata. L'OC est confrontée à une proximité des concurrents et un besoin de se rapprocher d'une clientèle de passage en tentant de la fidéliser. L'OCC est une grosse structure avec un volume de vente important bénéficiant de la proximité de l'hypermarché comme pôle d'attraction du client. Ces formes de proximité seront des critères exploités par les dirigeants selon le principe d'un management «sensoriel» (Torrès, 2009) permettant d'orienter leurs objectifs stratégiques selon leur perception de l'environnement.

C'est particulièrement important sur ce marché où il y a cette ambivalence entre la mission de santé publique et la nécessité d'avoir un commerce de détail rentable.

Ainsi, la vision du dirigeant-propriétaire sera le moteur de ses pratiques managériales. Cette construction mentale du futur visé (Carrière, 1990) est la principale source de différences entre les titulaires des officines étudiées. En effet, il a été constaté que les dirigeants de l'officine rurale (OR), en tant qu'hédonistes-artistes, ont la volonté de se rapprocher de leur rôle de professionnel de santé au détriment de celui de gestionnaire d'entreprise. Leurs pratiques de gestion y sont plus simples et ne doivent pas empiéter sur le temps consacré à la mission de santé. Pour la titulaire de l'officine de centreville (OC), ses pratiques de gestion sont également assez simples, mais pour une autre raison. En tant qu'alimentaire, c'est la survie qui mobilise toute son attention. La proximité concurrentielle l'étouffe et le dirigeant est obnubilé par la rentabilité de son officine. Les titulaires de l'officine de quartier (OQ) ont déjà une expérience de l'installation. Cette officine n'est pas une étape dans leur projet de carrière, mais la possibilité de façonner l'entreprise à leur image. En tant qu'hédonistes carriéristes, elles recherchent l'alliance entre la mission de santé de proximité et le développement de l'entreprise. Leurs pratiques de gestion sont plus développées et formalisées, mais délèguent peu pour s'y consacrer, car elles doivent être au comptoir pour être proches 
du client. Les titulaires de l'officine de centre commercial (OCC) peuvent s'appuyer sur une équipe importante et se consacrer au management de l'officine. En tant que carriériste, leur objectif pour faire face à l'incertitude du marché est de développer leur entreprise. Cela se traduit par une standardisation et une formalisation de leurs pratiques de gestion. Chacun de ces choix correspond à la réponse personnelle des dirigeants aux évolutions du marché.

Ainsi, une implication managériale importante pour cette recherche est que le fonctionnement de l'officine française ne répond pas à des standards de développement ou d'organisation comme n'importe quel commerce de détail. La volonté a été de montrer que le dirigeant en tant que propriétaire et professionnel libéral entend gérer son activité à sa façon. C'est sur ce plan que le management de proximité s'illustre le plus (Torrès, 2003). Ainsi, il semble important pour le pharmacien titulaire d'accepter que la réponse au marché ne soit pas de privilégier forcément le développement et l'expansion de l'activité, mais une approche adaptée à sa vision de son métier.

Les limites de cette recherche sont liées au petit nombre d'entreprises étudiées, nécessaire pour approfondir le sujet, mais qui restreint la portée des résultats. Elles laissent cependant entrevoir des perspectives intéressantes en augmentant le nombre de cas étudiés, dans un premier temps, en passant à une analyse quantitative, dans un second temps. Cela permettrait d'apporter des réponses au pharmacien sur les styles de management pour faire face aux évolutions de son marché et de dissiper certaines inquiétudes concernant l'avenir de son métier.

\section{Bibliographie}

AllianCE HEALTHCARE (2009), «Le carnet de route de votre installation », Document professionnel.

AMABILE, S. et M. GADILLE (2003), «Les NTIC dans les PME: stratégies, capacités organisationnelles et avantages concurrentiels », Revue française de gestion, vol. $3, \mathrm{n}^{\circ} 144$, p. 43-64.

BADOT, O. et J.-F. LEMOINE (2010), «Les stratégies d'innovation dans le commerce indépendant de proximité », Décisions Marketing, n 57, p. 63-66.

BARDIN, L. (2003), L'analyse de contenu, Paris, Presses universitaires de France.

BAYAD, M., M. GALLAIS et C. SCHMITT (2006), «Prescription et adoption des outils de gestion dans les PME: quels facteurs facilitateurs? », Congrès international francophone en entrepreneuriat et PME, Fribourg. 
BAYAD, M. et D. GARAND (1998), «Vision du propriétaire-dirigeant de PME et processus décisionnel: de l'image à l'action », Congrès international francophone de la PME, Metz.

Blanchet, A. et A. GotmAm (2005), L'enquête et ses méthodes: L'entretien, Paris, Armand Colin.

BOUTARY, M. (2008), «TIC et TPE: entre proximité et modernité», dans A. Jaouen et $\mathrm{O}$. Torrès (dir.), Les très petites entreprises: un management de proximité, Paris, Hermès Lavoisier, p. 57-80.

CAPPELLETTI, L. et D. KHOUATRA (2009), «L'implantation d'un système de contrôle de gestion au sein d'entreprises libérales: le cas des offices de notaires », Comptabilité Contrôle Audit, tome 7, vol. 1, mars, p. 129-146.

CARRIÈRE, J.-B. (1990), «La vision stratégique en contexte de PME: cadre théorique et étude empirique», Revue internationale PME, vol. 3, nos 3-4, p. 301-325.

CONDOR, R. et K. REBUT (2008), «Déterminants et modes opératoires du contrôle de gestion dans les PME: une approche qualitative comparative», Congrès international francophone en entrepreneuriat et PME, Louvain-la-Neuve.

COSSETTE, P. (1996), «La vision stratégique du propriétaire-dirigeant de PME: étude de cartographie cognitive» Revue internationale PME, vol. 9, n 1, p. 123-142.

DÁVILA, A. et G. FOSTER (2005), «Management accounting systems adoption decisions: evidence and performance implications from early-stage/startup companies », The Accounting Review, vol. 80, n 4, p. 1039-1068.

DEBARGE, O. (2011). «La distribution au détail du médicament au sein de l'Union Européenne: croisement entre santé et commerce», Revue internationale de droit économique, tome XXV, vol. 2, p. 193-238.

DÉClARATION DU ROI (1777), Portant règlement pour les professions de la pharmacie \& de l'épicerie à Paris, donnée à Versailles le 25 avril 1777, registrée en Parlement le 13 mai 1777, Paris, P.G. Simon, 4 p.

EISENHARDT, K. (1989), «Building theories from case study research », Academy of Management Review, vol. 14, n 4, p. 532-550.

FONROUGE, C. (2002), «L'entrepreneur et son entreprise: une relation dialogique», Revue française de gestion, $\mathrm{n}^{\circ} 138$, p. 145-158.

GAVARD-PERret, M.-L., D. GotTElAnd, C. HAON et A. Jolibert (2008), Méthodologie de la recherche: réussir son mémoire ou sa thèse en sciences de gestion, Paris, Pearson Éducation France.

GERMAIN, C. (2006), «Le pilotage de la performance dans les PME en France: une comparaison des pratiques de tableaux de bord des organisations familiales et des filiales », Revue internationale PME, vol. 19, n 1, p. 69-94.

HLADY-RISPAL, M. (2002), La méthode des cas: application à la recherche en gestion, Bruxelles, De Boeck.

IMPACT PHARMACIEN (2009), «Comment se dessine la pharmacie de demain», $n^{\circ} 232$, octobre. 
JAOUEN, A. (2008), «Introduction », dans A. Jaouen et O. Torrès (dir.), Les très petites entreprises: un management de proximité, Paris, Hermès Lavoisier, p. 19-24.

JAOUEN, A. (2010), «Typologie de dirigeants de très petite entreprise », Journal of Small Business and Entrepreneurship, vol. 23, nº 1, p. 113-152.

JAOUEN, A. et N. TESSIER (2008), «Effet de grossissement et proximité dans les pratiques de GRH des très petites entreprises ", dans A. Jaouen et O. Torrès (dir), Les très petites entreprises: un management de proximité, Paris, Hermès Lavoisier, p. 143-165.

Le pharmacien de France (2011), «L'heure du bilan», avril, n 1229.

MAHÉ DE BOISLANDELlE, H. (1996), «Effet de grossissement et management des ressources humaines en $\mathrm{PME}$, Congrès international francophone en entrepreneuriat et PME, Trois-Rivières.

MARCHESNAY, M. (1992), «La PME: une gestion spécifique?», Problèmes économiques, mai, $\mathrm{n}^{\circ} 2.276$, p. 26-32.

MARCHESNAY, M. (2003), «La petite entreprise: sortir de l'ignorance», Revue française de gestion, vol. 3, n 144 , p. 107-118.

MARCHESNAY, M. (2008), «Trente ans d'entrepreneuriat et PME en France, naissance, connaissance, reconnaissance », Revue internationale PME, vol. 21, $\mathrm{n}^{\circ} 2$, p. $145-168$.

MARTIN, J. (2001), «Recherche biomédicale: intérêts privés et intérêt public», Santé publique, vol. 13, no 1, p. 89-93.

Messeghem, K. et S. SAMMUT (2011), L'entrepreneuriat, Paris, Éditions EMS.

MEYSSONNIER, F. et C. ZAWADZKI (2008), «L'introduction du contrôle de gestion en PME», Revue internationale PME, vol. 21, n 1, p. 69-92.

MOINIER, X. (2009), «L'enseigne de pharmaciens: une spécificité au cœur de la distribution », Décisions Marketing, n 53, janvier-mars, p. 7-17.

MOMAS, I. (2009), «La santé publique et le pharmacien: des enjeux primordiaux de formation», Annales pharmaceutiques françaises, n 67, p. 25-31.

NOBRE, T. (2001), «Méthodes et outils de contrôle de gestion dans les PME», Finance contrôle stratégie, vol. 4, $\mathrm{n}^{\circ}$ 2, juin, p. 125-143.

NOGUERA, F. et J. LARTIGAU (2009), «De la prospective à la gestion prévisionnelle des métiers et des compétences dans la fonction publique hospitalière enjeux et perspectives », Management \& Avenir, vol. 25, p. 290-314.

ORDRE NATIONAL DES PHARMACIENS (2011), «Démographie des pharmacies», document Internet.

PARADAS, A. (1996), «Typologies du dirigeant et styles de gestion des ressources humaines dans la PME», Congrès international francophone sur la PME, Trois-Rivières.

Pharmacien manager (2011), «Parapharmacie la fin du mythe», $\mathrm{n}^{\circ} 105$, mars.

Rapport de la Cour des comptes sur le financement de la sécurité sociale (2008), «Chapitre VI», septembre, p. 193-221. 
RAPPORT N ${ }^{\circ} 48$ (2008), «La prescription, la consommation et la fiscalité des médicaments », Assemblée Nationale, 30 avril, 503 p.

REIJONEN, I. et R. KOMPPULA (2007), «Perception of success and its effect on a small firm performance», Journal of Small Business and Enterprise Developpement, vol. 14, no 4, p. 689-701.

REYES, G. (2011), «Les mutations du métier de pharmacien titulaire: le cas d'officines de centre commercial», Management \& Avenir, n 46, p. 79-99.

SANDINO, T. (2007), «Introducing the first management control systems: evidence from the retail sector», The Accounting Review, vol. 82, n 1, p. 265-293.

SCHMITT, C., P.-A. JULIEN et R. LACHANCE (2002), «Pour une lecture des problèmes complexes en PME: approche conceptuelle et expérimentation », Revue internationale PME, vol. 15, n 2, p. 35-62.

SIMON, P.(2010), «De nouvelles organisations et pratiques de soins par télémédecine», Médecine des maladies métaboliques, vol. 4, n 3, p. 1-6.

Silvestre, H. et R. GOUJET (1996), «Lisibilité de l'environnement, management stratégique: éléments de recherche sur les PMI», Revue internationale PME, vol. 9, no 1, p. 61-78.

SMIDA, A. et R. CONDOR (2002), «Interaction entre vision, intention et anticipation chez les dirigeants de petites entreprises », Gestion, vol. 26, n 4 , p. 12-22.

SMITH, N.R. (1967), «The entrepreneur and his firm: the relationship between type of man and type of company», Occasional Papers, Bureau of Business Research, Michigan State University, Michigan, 109 p.

TABUTEAU, D. (2010), «Les nouveaux défis de santé: pouvoirs publics et professions de santé», Les tribunes de la santé, n 26, p. 103-121.

TORRÈS, O. (2003), «Petitesse des entreprises et grossissement des effets de proximité», Revue française de gestion, $\mathrm{n}^{\circ}$ 144, mai-juin, p. 119-138.

TORRÈS, O. (2009), «La recherche en PME an V.I.T.R.I.O.L.», Économie et Société, série $\mathrm{K}, \mathrm{n}^{\circ} 20$, p. 342-362.

TORRÈS, O. et G. GUÉGUEN (2008), «Incidence de la loi proxémique sur la perception de l'incertitude des PME», Revue internationale PME, vol. 21, nº 1, p. 93-115.

VAN CAILliE, D. (2003), «L'exercice du contrôle de gestion en contexte PME: étude comparée des cas français, canadien et belge », Congrès annuel de l'Association francophone de comptabilité, Louvain-la-Neuve.

WACHEUX, F. (1996), Méthodes qualitatives en recherche en gestion, Paris, Économica.

YIN, R.K. (2008), Case Study Research: Design and Methods, Los Angeles, Sage Publications. 